\title{
Traceably calibrated scanning Hall probe microscopy at room temperature
}

\author{
Manuela Gerken ${ }^{1}$, Aurélie Solignac ${ }^{2}$, Davood Momeni Pakdehi ${ }^{1}$, Alessandra Manzin ${ }^{3}$, \\ Thomas Weimann $^{1}$, Klaus Pierz ${ }^{1}$, Sibylle Sievers ${ }^{1}$, and Hans Werner Schumacher ${ }^{1}$ \\ ${ }^{1}$ Physikalisch-Technische Bundesanstalt (PTB), Bundesallee 100, 38116 Braunschweig, Germany \\ ${ }^{2}$ SPEC, CEA, CNRS, Université Paris-Saclay, CEA Saclay, 91191 Gif-sur-Yvette, CEDEX, France \\ ${ }^{3}$ Istituto Nazionale di Ricerca Metrologica, 10135 Turin, Italy \\ Correspondence: Manuela Gerken (manuela.gerken@ptb.de) and Sibylle Sievers (sibylle.sievers@ptb.de)
}

Received: 11 June 2020 - Revised: 31 August 2020 - Accepted: 2 October 2020 - Published: 13 November 2020

\begin{abstract}
Fabrication, characterization and comparison of gold and graphene micro- and nanoscale Hall sensors for room temperature scanning magnetic field microscopy applications are presented. The Hall sensors with active areas from $5 \mu \mathrm{m}$ down to $50 \mathrm{~nm}$ were fabricated by electron-beam lithography. The calibration of the Hall sensors in an external magnetic field revealed a sensitivity of $3.2 \mathrm{mV} \mathrm{A}^{-1} \mathrm{~T}^{-1} \pm 0.3 \%$ for gold and $1615 \mathrm{~V} \mathrm{~A}^{-1} \mathrm{~T}^{-1} \pm 0.5 \%$ for graphene at room temperature. The gold sensors were fabricated on silicon nitride cantilever chips suitable for integration into commercial scanning probe microscopes, allowing scanning Hall microscopy (SHM) under ambient conditions and controlled sensor-sample distance. The height-dependent stray field distribution of a magnetic scale was characterized using a $5 \mu \mathrm{m}$ gold Hall sensor. The uncertainty of the entire Hall-sensor-based scanning and data acquisition process was analyzed, allowing traceably calibrated SHM measurements. The measurement results show good agreement with numerical simulations within the uncertainty budget.
\end{abstract}

\section{Introduction}

High-resolution quantitative magnetic field measurements at the micrometer scale are increasingly important for research and development in areas like magnetic sensors and magnetic positioning. However, the characterization of microscale magnetic structures entails new challenges for magnetic stray field measurement techniques, since the generated magnetic stray fields locally change their direction on the nanometer range, and the field amplitude decreases rapidly with an increasing distance to the sample surface. Thus, suitable magnetic sensors not only need to be small to avoid averaging over different stray field directions but also must be precisely positioned close to the sample.

Here, micro- and nanoscale Hall sensors for traceable scanning Hall probe microscopy (SHPM) are characterized, and one technique to integrate them into a commercial atomic force microscope (AFM) is presented. AFM-based SHPM (Sandhu et al., 2004; Shaw et al., 2016; Sonusen et al., 2014) (AFM-SHPM) has certain advantages compared to other magnetic imaging techniques. In comparison to magnetic force microscopy (Hu et al., 2018; Kazakova et al., 2019), AFM-SHPM can be considered as being noninvasive due to the use of nonmagnetic materials and a neglectable magnetic field produced by the supply current. A measurement directly generates quantitative results albeit with reduced spatial resolution. It is applicable to a broader field range than magneto-optical indicator film techniques (Kabanov et al., 2005; McCord, 2015), which are limited by the saturation field of the sensor film. Unlike magneto-resistive sensors (Costa et al., 2015; Takezaki and Sueoka, 2008), Hall probes show excellent linearity without hysteresis and measure a well-defined field component perpendicular to the sensor plane. Furthermore, SHPM enables a low sample-probe distance and therefore a spatial resolution limited by the sensor size only. A three-dimensional mapping of the stray field can be performed by repeating the two-dimensional plane scans at defined heights (Dede et al., 2016), and first commercial systems employing submicron Hall sensors for room 
temperature (RT) measurements are available (NanoMagnetics Instruments, 2017a, b). Concerning the choice of the Hall sensor material, different material classes have been considered: standard Hall sensor devices are typically based on semiconductor technology. They show an outstanding performance at low temperatures (Hicks et al., 2007), and micronsized sensors also work at room temperature (Chenaud et al., 2016; Pross et al., 2005; Shaw et al., 2016). In contrast, nanoscale semiconductor Hall sensors show a weak signalto-noise $(\mathrm{S} / \mathrm{N})$ ratio at RT. Besides the semi-metal bismuth (Sandhu et al., 2004), graphene (Ciuk et al., 2019; Dauber et al., 2015; Huang et al., 2014; Sonusen et al., 2014; Tang et al., 2011) has the potential to bridge this gap. Semi-metallic graphene can be prepared with a low carrier density at RT, resulting in a high Hall coefficient. It could also enable the application of Hall sensors at high temperatures (Ciuk et al., 2019), in contrast to semiconductor sensing with a limited range of operational temperatures. Moreover, an active layer one atom thin prevents field averaging perpendicular to the sensor area, allowing high spatial resolution in the corresponding direction. Another option is the use of metals as Hall sensor materials. Here, in particular, gold is favorable due to its good electrical properties and its chemical stability. It offers a very stable carrier density, which is important for calibrated Hall measurements. A stable carrier density leads to a stable Hall coefficient and thus to a low uncertainty of the Hall sensitivity. Its carrier density is widely insensitive to surface contaminations as well as to the temperature, in contrast to semiconductors. Gold is simple to manage in the fabrication process. The fabrication is cheaper and less timeconsuming than for semiconductor-based Hall sensors. The substrate material can be chosen flexibly due to the simple deposition process of gold. This enables the fabrication of gold Hall sensors on cantilever tips and consequently the performance of AFM-SHPM. Strain induced in the sensor due to the cantilever oscillation has no influence on the electronic structure and thus on the Hall signal, whereas this cannot be neglected for semiconductors. Additionally, it was reported that gold Hall sensors with sizes below $500 \mathrm{~nm}$ have a better noise figure at RT than sensors based on two-dimensional electron gases (Novoselov et al., 2003).

In this work, the fabrication and investigation of gold- and graphene-based micro- and nanoscale Hall sensors with respect to SHPM applications are presented. The sensor sensitivity and stability are characterized and discussed. Gold Hall sensors are fabricated on silicon nitride ( $\mathrm{SiN}$ ) cantilever chips suitable for AFM-SHPM. After traceable calibration, they allow quantitative stray field measurements of magnetic scales with a resolution of a few microns. The uncertainty budget of the measurements is discussed, and the setup is validated by comparing the measurement results to stray field and Hall voltage signal calculations.

\section{Fabrication of Hall sensors}

The Hall sensor cross-structures with active square areas from $5 \mu \mathrm{m} \times 5 \mu \mathrm{m}$ down to $50 \mathrm{~nm} \times 50 \mathrm{~nm}$ were fabricated using electron-beam lithography (EBL). The substrate of the gold sensors consists of a silicon wafer covered on both sides with a $1 \mu \mathrm{m}$ thick SiN layer deposited by low-pressure chemical vapor deposition. The inset of Fig. 1a shows a gold crossshaped Hall sensor that is produced through two lithography steps. First, the Hall cross defining the sensor's active area is structured, after electron beam deposition of the metals, by EBL and lift-off through a PMMA resist. The active sensor material consists of a $5 \mathrm{~nm}$ titanium adhesion layer followed by a $30 \mathrm{~nm}$ gold layer. In the second step, an additional $50 \mathrm{~nm}$ gold layer is deposited to support the outer extended contact regions. After fabrication of the Hall sensors, cantilever chips with Hall sensors on the tips of the cantilevers, as depicted in Fig. 1, are fabricated out of the SiN wafer by optical lithography and etching. The shape of the cantilever is defined by an aluminum (Al) mask, resistant to the $\mathrm{SiN}$ final reactive ion etching (RIE). Windows are opened in the $\mathrm{SiN}$ on the backside by RIE, allowing the wet etching of silicon under the cantilever region with potassium hydroxide $(\mathrm{KOH})$. The cantilevers now stand up on windows of $1 \mu \mathrm{m}$ thick SiN and are protected by an $\mathrm{Al}$ mask. Cantilevers are then released by performing the final top RIE etching of the $\mathrm{SiN}$, and the $\mathrm{Al}$ mask is dissolved in a photoresist developer basic solution. By careful alignment of the Hall sensor and cantilever fabrication processes, a Hall sensor positioning close to the cantilever tip is possible. The geometrical dimensions are visualized in Fig. $1 \mathrm{~b}$ and c. For the $5 \mu \mathrm{m}$ sensors a minimal distance between the sensor center and triangular cantilever tip of $20 \mu \mathrm{m}$ can be achieved and for the $50 \mathrm{~nm}$ ones a distance of $2.4 \mu \mathrm{m}$.

The graphene samples were grown on silicon carbide (SiC) (0001) substrates with a size of $5 \mathrm{~mm} \times 10 \mathrm{~mm}$ using a so-called polymer-assisted sublimation growth technique (Kruskopf et al., 2016; Momeni Pakdehi et al., 2018, 2019). The high morphological and electronic homogeneity of the graphene samples utilizes scalable realization of Hall sensors on true two-dimensional carbon sheets without bilayer inclusions. The graphene Hall sensors were patterned with EBL and AC plasma-etching through a resist mask. The fabrication of graphene Hall cross-structures requires four steps. Initially, the electrical contacts are defined by depositing $\mathrm{Ti} / \mathrm{Au}(10 / 30 \mathrm{~nm})$ layers in a lift-off process. In the next step, the monolayer graphene is structured by plasma etching through an EBL resist mask, leading to well-defined geometry and sizes of the small active area as shown in Fig. 2. The finger-shaped contact structure produces a long contact line between graphene and gold in the plane, which enables an efficient charge transfer and reduces the contact resistance (Göktaş et al., 2008; Nagashio et al., 2010; Weis and von Klitzing, 2011). Finally, the outer contact areas and leads are defined using a $50 \mathrm{~nm}$ gold layer. To avoid environmental in- 
(a)

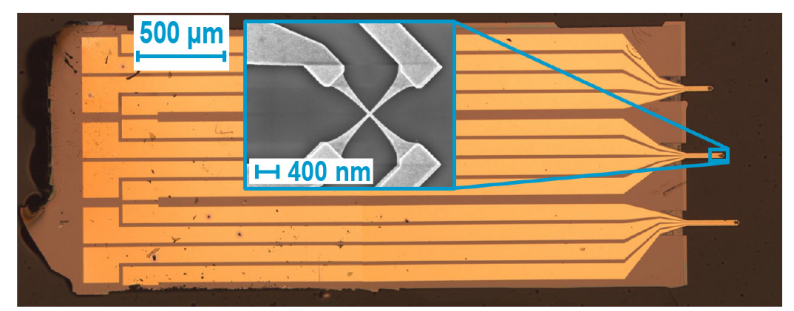

(d)

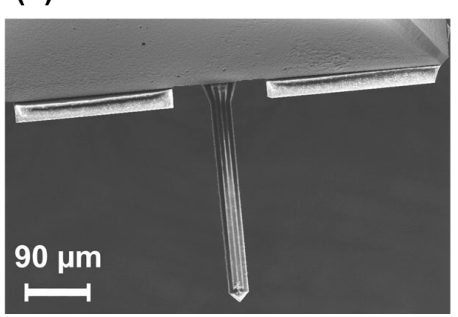

(b)

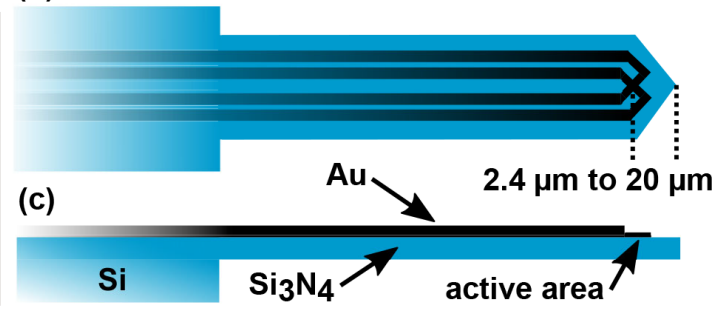

Figure 1. (a) Microscopy image of a $3.4 \mathrm{~mm} \times 1.5 \mathrm{~mm}$ cantilever chip with three gold Hall sensors for integration into commercial scanning probe microscopy systems. The inset shows a scanning electron microscopy image of a $50 \mathrm{~nm}$ gold Hall cross. (b) Top-view sketch of the Hall sensor on the cantilever. The distance between the active area and the triangular tip of the cantilever depends on the size of the sensor, so that the smaller sensors can be positioned closer to the tip. (c) Side view of the cantilever. Dimensions are exaggerated for the sake of visibility (the cantilever is $1 \mu \mathrm{m}$ thick). (d) SEM image of the cantilever from the backside. The gold contact lines are visible through the $1 \mu \mathrm{m}$ thin SiN layer.

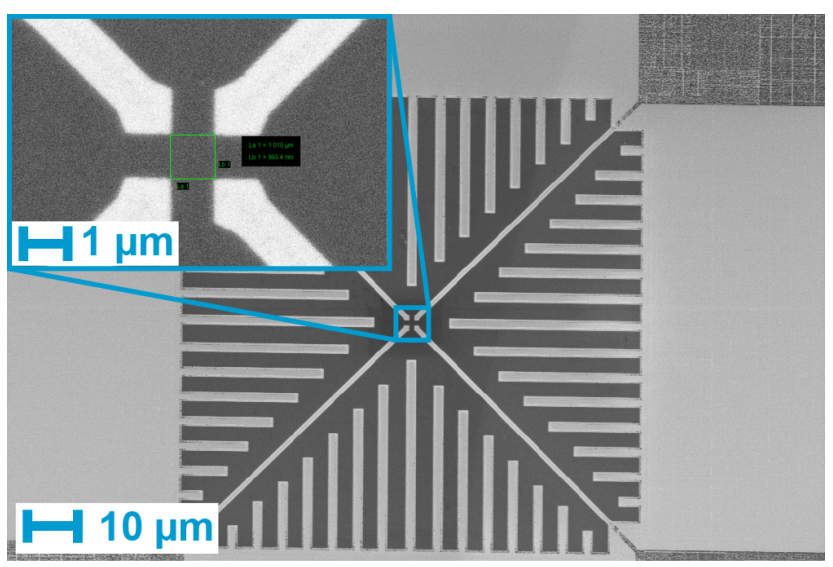

Figure 2. Scanning electron microscopy image of a $1 \mu \mathrm{m}$ graphene Hall cross and the contact region before deposition of the final gold layer. The inset shows a magnification of the cross area.

fluences, especially the rapid change of the carrier density by surface absorption on the graphene, the graphene sample was encapsulated with $50 \mathrm{~nm}$ co-polymer. Note that within this work the graphene sensors were only fabricated on $\mathrm{SiC}$ wafer dies. The fabrication of graphene sensors on cantilevers will be the subject of future studies.

\section{Characterization of the Hall sensors}

For the Hall sensor calibration, an electromagnet driven by a Kepco power supply with a pole shoe diameter of $92 \mathrm{~mm}$ was used to provide a spatially homogeneous magnetic flux density of up to $450 \mathrm{mT}$ at a pole shoe distance of $18.5 \mathrm{~mm}$. The operation current for the Hall sensors was generated by a Keithley SourceMeter 2400. The Hall voltage was measured with a Keithley Nanovoltmeter 2182A. During the Hall sensor calibration, the magnetic flux density was simultaneously measured with a traceably calibrated commercial Hall probe FH55 from Magnet-Physik Dr. Steingroever GmbH. As a consequence, the Hall sensor calibration is traceable to the SI units when considering and listing all uncertainty contributions, as presented in Sect. 6.

The typical output from the characterization of graphene and gold sensors is presented in Fig. 3. The Hall voltage was measured as a function of the magnetic flux density $B$ and corrected for the offset. Both sensors show a linear dependence of the Hall voltage $V_{\text {Hall }}$ on $B$ as expected from $V_{\text {Hall }}=I \times B /(n \times e \times t)$, where $I$ is the supply current, $n$ is the electron density, $e$ is the electron charge and $t$ is the thickness of the active layer. For the $5 \mu \mathrm{m}$ gold Hall sensor operated at $10 \mathrm{~mA}$, the output is in the microvolt $(\mu \mathrm{V})$ range for $B$ between -150 and $150 \mathrm{mT}$. This leads to a sensitivity of $3.2 \mathrm{mV} \mathrm{A}^{-1} \mathrm{~T}^{-1} \pm 0.3 \%$. For the same field range, the Hall voltage of the $500 \mathrm{~nm}$ graphene sensor is in the millivolt $(\mathrm{mV})$ range using an operating current of $50 \mu \mathrm{A}$. Fitting the data reveals a sensitivity of $1615 \mathrm{~V} \mathrm{~A}^{-1} \mathrm{~T}^{-1} \pm 0.5 \%$. The 


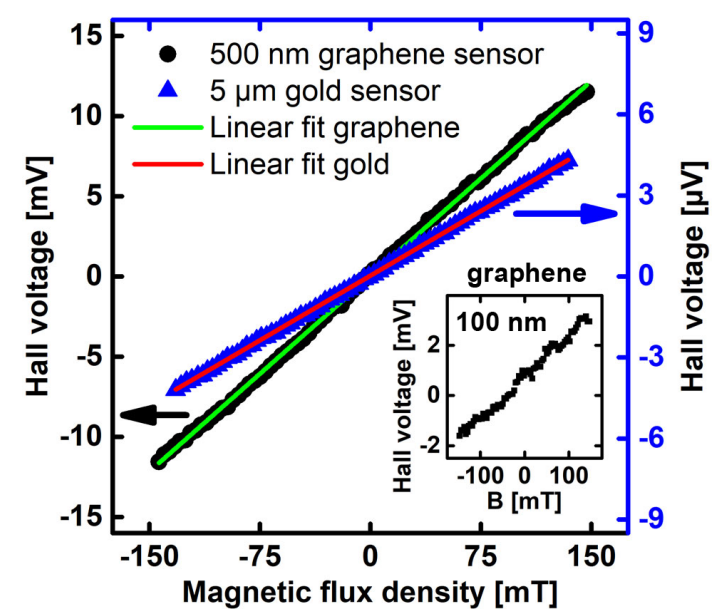

Figure 3. Typical calibration curves of gold and graphene Hall sensors. For both measurements, the offset was subtracted from the Hall voltage. The $500 \mathrm{~nm}$ graphene Hall sensor was operated at $50 \mu \mathrm{A}$ and exhibited a sensitivity of $1615 \mathrm{~V} \mathrm{~A}^{-1} \mathrm{~T}^{-1} \pm 0.5 \%$. A supply current of $10 \mathrm{~mA}$ was used for the $5 \mu \mathrm{m}$ gold sensor. The sensitivity extracted from the fitted result is $3.2 \mathrm{mV} \mathrm{A}^{-1} \mathrm{~T}^{-1} \pm 0.3 \%$. The inset shows the calibration result of a $100 \mathrm{~nm}$ graphene sensor operated at $10 \mu \mathrm{A}$. The sensitivity was determined to $1649 \mathrm{~V} \mathrm{~A}^{-1} \mathrm{~T}^{-1} \pm 1.17 \%$.

sensitivity of the graphene sensor is 6 orders of magnitude higher due to the lower carrier density of graphene in comparison to gold. Similar results were observed in the measurements on several other $5 \mu \mathrm{m}$ large Hall sensors. The mean sensitivity of all gold sensors is $3.1 \mathrm{mV} \mathrm{A}^{-1} \mathrm{~T}^{-1}$, with a maximum deviation of $0.2 \mathrm{mV} \mathrm{A}^{-1} \mathrm{~T}^{-1}$ within the sensors in this study.

Moreover, the time stability as well as fabrication reproducibility of the sensors were investigated. To this end, the sensors were frequently characterized within 1 year and compared with nominally identical sensors from different batches. For the gold sensors, the long-term stability was very high, with a deviation over time below $0.6 \%$. Graphene sensors showed sensitivity deviations of up to $9.3 \%$ from one day to another. The direction of the deviations is random; there is no systematic drift present. This might be caused by charges attaching and detaching on the co-polymer which act like a gate and thus influence the carrier density in the graphene. Also, the overall variation in sensitivity was larger for graphene sensors, ranging from 500 to $1700 \mathrm{~V} \mathrm{~A}^{-1} \mathrm{~T}^{-1}$ depending on the carrier density in the respective graphene material and actual sensor. Based on the Hall voltage deviation of measured data points from the expected value given by the linear characterization fit, a typical resolution of $2 \mathrm{mT}$ for gold sensors and $0.45 \mathrm{mT}$ for the graphene sensors is calculated. This resolution also includes, besides the sensor properties, influences and noise contributions from devices and cables in the circuitry. Noise measurements revealed a detectivity of $60 \mu \mathrm{T} \mathrm{Hz}^{-\frac{1}{2}}$ at $1 \mathrm{~Hz}$ for a graphene Hall cross at an operating current of $500 \mu \mathrm{A}$. The background noise of the system is $3 \mathrm{nV} \mathrm{Hz}^{-\frac{1}{2}}$. More details about the noise measurement system can be found in Nording et al. (2017) and Nording (2019). The property data are summarized in Table 1. Due to the small resistance of the gold Hall sensors, it was not possible to measure their noise characteristics. This also means that the noise properties of the electronics have a larger influence on the $\mathrm{S} / \mathrm{N}$ than the sensor itself. With the described measurement equipment, it was possible to calibrate gold sensors with active areas down to $1 \mu \mathrm{m}$. For $50 \mathrm{~nm}$ gold sensors on cantilevers, the background noise of the setup is larger than the expected Hall voltage of $1.5 \mathrm{nV}$ per $10 \mathrm{mT}$ at the operating current of $50 \mu \mathrm{A}$. Because of higher sensitivity and thus larger Hall voltage, graphene sensors with a size of $100 \mathrm{~nm}$ still show an overall linear dependence on the applied magnetic flux density, as shown in the inset of Fig. 3. The resolution is decreased to $8.5 \mathrm{mT}$ because of the growing impact of carrier fluctuations for smaller sensor sizes. Furthermore, the lower current supply leads to smaller Hall voltages; thereby the overall $\mathrm{S} / \mathrm{N}$ is reduced. This comparison of gold- and graphene-based Hall sensors emphasizes the benefits and drawbacks of metallic and semimetallic Hall sensors with the same small active areas and under consideration of the uncertainty budget.

\section{AFM-based SHPM - setup and measurement}

AFM-SHPM is realized by integration of the manufactured cantilever chips with gold Hall sensors into a commercial AFM (Nanoscope IIIa, Dimension 3000 scanner). The cantilevers have a typical resonance frequency of about $50 \mathrm{kHz}$ and can be used in standard tapping mode operation and thus in close contact with the sample surface. The cantilever oscillation leads to a vertical averaging of the field. However, for magnetic structures with features in the $100 \mu \mathrm{m}$ range, this averaging over typical cantilever amplitudes of a few tens of nanometers $(\mathrm{nm})$ is neglectable. As shown in Fig. 4a, the Hall sensors are positioned at the bottom side of the cantilever and close to its tip to achieve a small distance between the sensor and sample. This is significant for improving spatial resolutions in measuring nanostructures due to the fast decay of stray fields with increasing distance to the sample surface. The cantilevers are mounted at an angle of $10^{\circ}$ given by the cantilever holder. This leads to minimal measurement heights of 400 and $3366 \mathrm{~nm}$ for the $50 \mathrm{~nm}$ - and $5 \mu \mathrm{m}$-sized sensors, respectively, for an ideal alignment of the Hall sensor on the cantilever. The electrical connection to the Hall sensor is realized by bond wires from the cantilever chip to a printed circuit board (PCB) that is fixed to the cantilever holder. The current source, voltmeter and PCB are connected via soldered cables. To increase the scan area up to the millimeter range, additional piezo tables were added to the setup that allow the sample to be scanned with a fixed cantilever position. The Hall sensor was calibrated in the electromagnet be- 
Table 1. Properties of $5 \mu \mathrm{m}$ graphene and gold Hall sensors.

\begin{tabular}{lllll}
\hline Sensor material & Sensitivity & Resolution & Detectivity @ 1 Hz & Long-term deviation \\
\hline Gold & $2.9-3.3 \mathrm{mV} \mathrm{A}^{-1} \mathrm{~T}^{-1}$ & $2 \mathrm{mT}$ & - & $0.6 \%$ \\
SiC/graphene & $500-1700 \mathrm{~V} \mathrm{~A}^{-1} \mathrm{~T}^{-1}$ & $0.45 \mathrm{mT}$ & $60 \mu \mathrm{T} \mathrm{Hz}^{-\frac{1}{2}}$ & $9.3 \%$ \\
\hline
\end{tabular}

fore and after the AFM-SHPM measurement. As a test sample, a commercially available magnetic-scale SST250HFA04 from Sensitec was chosen. By the selection of this sample, the operation of AFM-SHPM can be demonstrated on a real and industrially relevant magnetic sample. Thin film conductors or coils, as known from magnetic force microscopy (MFM) calibrations (Corte-León et al., 2020), are not able to generate suitable stray fields of about $100 \mathrm{mT}$ on the micrometer scale. The commercial magnetic scale is made of a wet pressed strontium ferrite with a remanence magnetization of $M_{\mathrm{r}}=395 \mathrm{mT}$. The material was magnetized into alternating up and down magnetized stripes with a width of nominally $250 \mu \mathrm{m}$ and a length of several millimeters.

Figure $4 \mathrm{~b}$ displays the results of AFM-SHPM on the scale using a $5 \mu \mathrm{m}$ gold sensor on the shortest cantilever with a Hall sensitivity of $2.3 \mathrm{mV} \mathrm{A}^{-1} \mathrm{~T}^{-1} \pm 13 \%$, measured under an applied operating current of $1 \mathrm{~mA}$. Line scans with 10 repetitions each were performed at seven different measurement heights. The closest line scan to the sample was attained in the tapping mode and thus followed the sample topography at a distance of approximately $4 \mu \mathrm{m}$. The scans for higher distances were carried out at fixed heights ranging from 19 to $169 \mu \mathrm{m}$ (with $30 \mu \mathrm{m}$ intervals) by moving the sample with a 3 -axis piezo scanning system and fixed probe position. The sample has a granular structure and thereby height variations of around $10 \mu \mathrm{m}$ and locally tilted surface areas. Therefore, during the measurement in tapping mode, the z-piezo table was utilized to keep the sensor-sample distance in a range that is controllable by the AFM head. Geometrical features of the sample surface still influence the measurement result as indicated in Fig. 4c. The data sets of the two scan directions forward and backward as well as their average are compared. By averaging the two directions, systematic influences from the sample topography can be reduced. Furthermore, the granular and tilted surface of the sample leads locally to additional stray fields parallel to the sample which are also detected by the Hall sensor due to the cantilever tilt angle of $10^{\circ}$. However, this rather local contributions are expected to decay faster with increasing measurement height than the stray field generated by the overall magnetization pattern. The measurement was performed at a scan speed of $50.5 \mu \mathrm{m} \mathrm{s}^{-1}$. A total of 400 points were measured per line with an averaging time of $20 \mathrm{~ms}$. All plots in Fig. $4 \mathrm{~b}$ show the expected $500 \mu \mathrm{m}$ periodicity of the scale. The decay of the stray field amplitude with increasing distance to the sample is clearly visible in both actual measurement values and simulation results, as presented in the next paragraph. Fur- thermore, the cantilever and sensor appeared to be very robust, allowing the characterization of rather rough samples, as demonstrated in this study.

\section{Comparison with simulations}

Here, two modeling approaches are presented to validate the measurement procedure. The first one uses a Fourier transform method to calculate the $z$ component $B_{z}$ of the stray field produced by the magnetic scale. Assuming that the sample is perfectly parallel to the Hall sensor surface, and neglecting the $10^{\circ}$ cantilever tilt, the Hall sensor response is mainly dominated by the perpendicular component. From now on, this will be called the perpendicular or $z$ component in contrast to the in-plane components lying parallel to the sample surface. The calculated field profile is then compared to the experimental one, which is derived from the measured Hall voltage as $B=\left(V_{\mathrm{Hall}} \times n \times e \times t\right) / I$ after the application of offset corrections. In the following, the stray field simulation procedure is described. To simulate the perpendicular stray field component, the underlying sample magnetization has to be known. To this end, the magnetization is guessed from the measured Hall signal based on the following assumptions. (i) The transition between up and down magnetized poles can be found at zero transitions of the Hall signal after a performed offset and drift correction. (ii) The magnetization pattern of the scale, therefore, can be found by discrimination between areas with positive and negative Hall voltages. (iii) Areas with $+V_{\text {Hall }}\left(-V_{\text {Hall }}\right)$ have purely perpendicular and, over the thickness, homogenous magnetization of $+M_{\mathrm{r}}\left(-M_{\mathrm{r}}\right)$. To account for the pole writing process, the step-like transitions are then additionally smoothed as visualized in Fig. 5a. For the stray field calculation, a transfer function (TF) approach (Hug et al., 1998; van Schendel et al., 2000) was pursued due to its numerical simplicity. It can be shown that in a partial Fourier space of only the in-plane spatial components, as for the transformation from $(x, y, z)$ to $\left(k_{x}, k_{y}, z\right)$, the calculation of the stray field above a perpendicular magnetization distribution can be performed through a multiplication by a $M_{z}$ to $B_{z} \mathrm{TF}$.

$\mu B_{z}(\boldsymbol{k}, z)=\mu_{0} M_{z}(\boldsymbol{k}) \times \frac{\left(1-e^{-k d}\right) e^{k z}}{2}$

The stray field of the scale at measurement height $z$ was calculated for a magnetic layer with an assumed thickness of $d=75 \mu \mathrm{m}$. Similarly, the impact of the finite sensor di- 
(a)

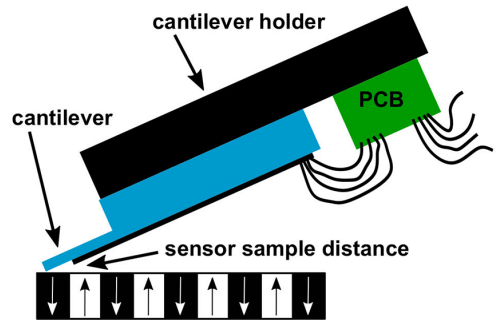

(b)
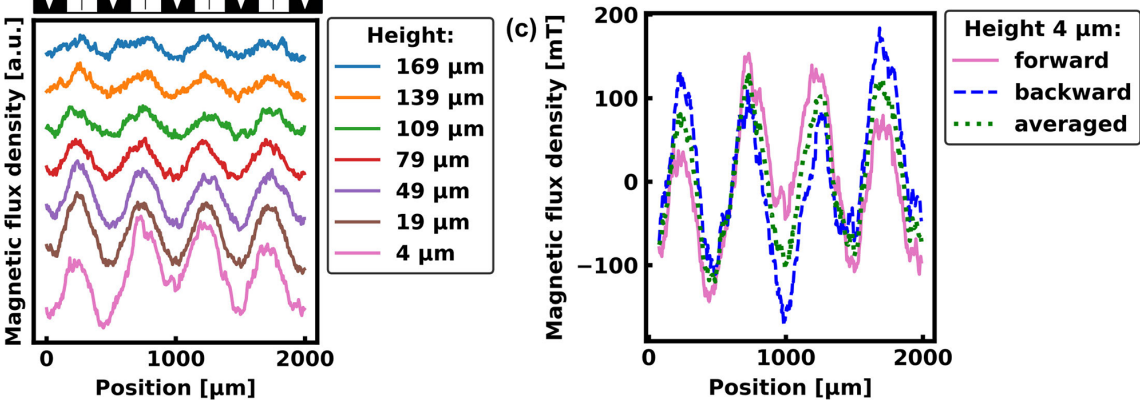

Figure 4. (a) Schematic of the AFM-SHPM measurement principle with electrical connections to the sensor and the minimal sensor-sample distance. (b) The height-dependent SHPM of a magnetic scale with $250 \mu \mathrm{m}$ pole width is shown. A $5 \mu \mathrm{m} \times 5 \mu \mathrm{m}$ gold Hall sensor scanned one line of the sample 10 times for each height. The measurement at $4 \mu \mathrm{m}$ was performed in tapping mode so that the cantilever tip was in contact with the sample surface. Data from the different measurement heights were shifted to avoid overlapping. (c) Influence of the scan direction for the measurement in tapping mode. The data of the forward (backward) line are based on 10 scans from left to right (right to left).
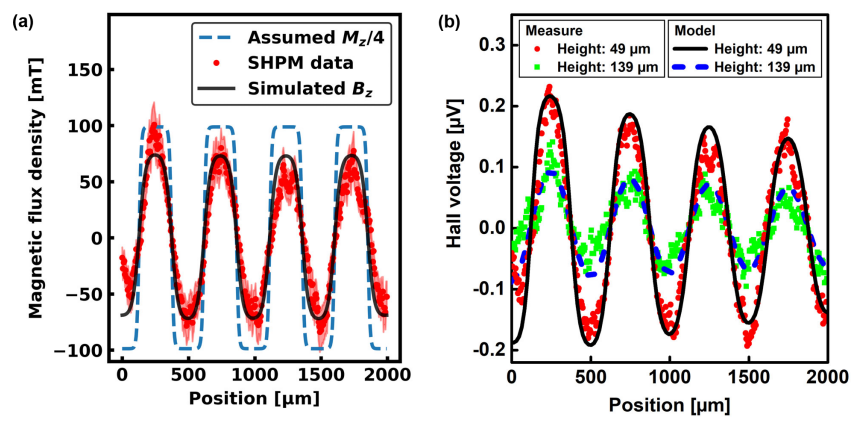

Figure 5. (a) Traceable SHPM data with uncertainty budget of scale SST250HFA-04 at a measurement height of $49 \mu \mathrm{m}$ compared to simulated stray field values from the assumed magnetization distribution. (b) Comparison of experimental and calculated Hall voltage signal for measurement heights of 49 and $139 \mu \mathrm{m}$. The calculation is done with the second modeling approach, introducing an angular misalignment of about $1^{\circ}$ between the magnetic sample and the Hall device to explain the drift during scanning.

mensions might be considered by introducing a multiplication by an appropriate sensor sensitivity TF. However, for the relatively slowly varying field of the scale with $250 \mu \mathrm{m}$ pole width, this is expected to have a minor impact and was therefore neglected. Inverse discrete Fourier transformation was used to obtain the value of the perpendicular component plotted in Fig. 5a for each SHPM measured point in real space.

A good agreement between the measured stray field and simulated data was obtained, giving evidence for the validity of the measured quantitative magnetic field distribution, as shown exemplarily in Fig. 5a for the sensor-sample distance of $49 \mu \mathrm{m}$. The simulation confirms the measurement results very well in terms of maximal and minimal magnetic flux density as well as spatial periodicity. However, the drift and slight decrease in amplitude cannot be explained by this stray field simulation. One reason would be an unstable temperature during the measurement and thus a drift of the offset voltage. Another explanation would be an angular misalignment of the sample if it is not flat or placed perfectly horizontally on the table. Moreover, the implementation of the cantilever chip in the AFM with a $10^{\circ}$ canting angle, resulting in a canted Hall sensor with respect to the sample surface, would lead to an asymmetric signal. As a proof, a second modeling approach was implemented, in which the Hall voltage signal due to magnetic-scale scanning is numerically calculated, considering an angular misalignment of about $1^{\circ}$ between the magnetic sample and the Hall device. The spatial distribution of the electric potential $\phi$ within the sensor is derived from the finite element solution (Manzin and Nabaei, 2014) of the following equation:

$\nabla \cdot[\ddot{\sigma}(\boldsymbol{r}) \nabla \phi(\boldsymbol{r})]=0$,

where $\ddot{\sigma}(\boldsymbol{r})$ is the conductivity tensor,

$\ddot{\sigma}(\boldsymbol{r})=\frac{\mu n e}{1+\left[\mu B_{\perp}(\boldsymbol{r})\right]^{2}}\left[\begin{array}{cc}1 & \mu B_{\perp}(\boldsymbol{r}) \\ -\mu B_{\perp}(\boldsymbol{r}) & 1\end{array}\right]$. 
In Eq. (3) $\mu$ is the electron mobility, assumed equal to $8.7 \times 10^{-4} \mathrm{~m}^{2} \mathrm{~V}^{-1} \mathrm{~s}^{-1}$ from the four-point resistance measurement, $n=1.92 \times 10^{21} \mathrm{~m}^{-2}$, and $B_{\perp}$ is the orthogonal component to the sensor of the stray field from the scale below, which also includes the component of $B$ parallel to the sample surface, due to the sensor-sample relative angular orientation. The formulation is completed by the boundary conditions in correspondence of the current and voltage contacts.

The stray field from the scale, which is discretized in $N$ $10 \mu \mathrm{m}$ size hexahedra with imposed uniform magnetization, is calculated as

$\boldsymbol{B}(\boldsymbol{r})=\frac{\mu_{0}}{4 \pi} \sum_{e=1}^{N} \int_{\partial \Omega_{e}} \boldsymbol{M}\left(\boldsymbol{r}_{e}\right) \cdot \boldsymbol{n}_{e} \frac{\left(\boldsymbol{r}-\boldsymbol{r}_{e}\right)}{\left\|\boldsymbol{r}-\boldsymbol{r}_{e}\right\|^{3}} d s$,

where $\partial \Omega_{e}$ is the surface of the $e$ th hexahedron with the normal unit vector $\boldsymbol{n}_{e}$ and barycenter with vector position $\boldsymbol{r}_{e}$ (Nabaei et al., 2013).

The drift effect in the measured Hall voltage signal is well reconstructed by the numerical results, which also support the validity of the linear dependence of $V_{\text {Hall }}$ on $B$ for all the scanning points, due to the large width of the pole scale with respect to the Hall cross size. The agreement with experimental results is highlighted in Fig. 5b for an average sensorsample distance of 49 and $139 \mu \mathrm{m}$. The peaks reduce in amplitude during scanning as a consequence of the increase in the sensor-sample distance.

For further verification, the behavior of the stray field with an increasing distance to the sample surface, as shown in Fig. 4, was quantitatively analyzed. Therefore, the maximal measured stray field amplitudes over the poles for each measurement height were compared in Fig. 6 with the values expected from simulations with the first approach. For the two largest measurement heights and corresponding two lowest expected magnetic flux densities, systematic uncertainties from evaluating the extrema have a more significant influence on the result due to an enlarged contribution of noise. However, for all measurements, the simulation result overlaps with the uncertainty squares of the data points. More details about the uncertainty range are given in the next paragraph. From these results, the validity of the quantitative AFM-SHPM method using a gold sensor is concluded.

\section{Evaluation of uncertainty budget}

A traceable scanning Hall sensor calibration obligatorily requires an analysis of all contributions of the entire scanning and read-out process to the uncertainty and a statement of their values. Five major contributions to the net measurement uncertainty are as follows: (i) the Hall sensor itself, for which its stability, sensitivity, offset, temperature dependence and noise must be considered; (ii) the Hall sensor calibration via the electromagnets' magnetic field homogeneity, stability and repeatability; (iii) the Hall sensor driving and read-

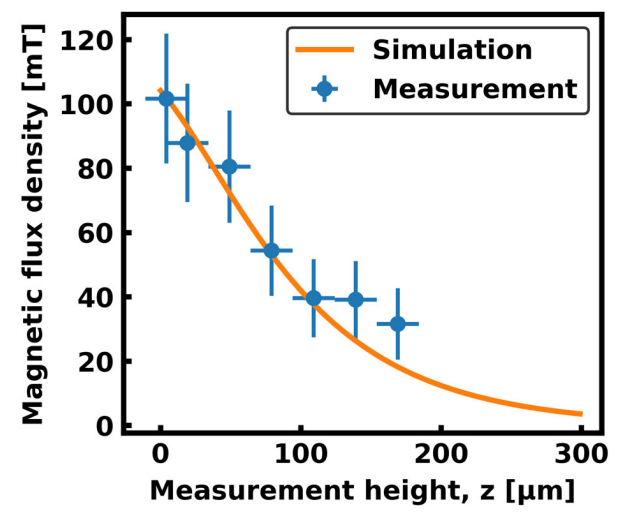

Figure 6. Simulated stray field decay with increasing measurement height over the middle of a pole. The data points are generated from the measurements shown in Fig. 4 by averaging the absolute values of the three minima and four maxima.

out electronics, including the stability of the current source and the voltmeter noise as well as thermoelectric voltages; (iv) the positioning accuracy of the scanning system; and (v) the influence of the sensor on the sample, for example in terms of the magnetic stray field generated by the supply current. The multiplicity of uncertainty sources and the fact that standard uncertainty analysis is not sufficient for linear regression tasks (Klauenberg et al., 2015), as used in the Hall sensor calibration, rule out a conventional uncertainty propagation calculation. Therefore, the uncertainties of the main contributions were analyzed separately to evaluate their impact on the measurement.

By repeated sensor calibrations and statistical analyses of the results, a calibration uncertainty for the sensor sensitivity of $13 \%$ was found. This uncertainty includes contributions from the Hall sensor itself (i), the applied magnetic flux density (ii) and the electronic components (iii). The characterization of several gold sensors, as shown above, leads to the expectation of lower sensitivity uncertainties for the gold sensors in general. The scanning system uncertainty (iv) is $7 \mathrm{mT}$ after the correction of drifts by offset subtraction. This was evaluated from 10 repeated line scans. The different measurement heights were realized by an additional $z$-piezo table that has a positioning accuracy of $15 \mu \mathrm{m}$. The stray field produced by the sensor supply current at the sample surface was estimated with the help of the Biot-Savart law to be $0.25 \mathrm{mT}(\mathrm{v})$. This has no influence on the sample characterized here and is neglectable for many other applications. Combining all contributions leads to an extended uncertainty of $\pm(7 \mathrm{mT}+13 \%)$ for the SHPM using the amplification factor $k=2$.

\section{Conclusion}

In summary, SiN-based AFM cantilevers equipped with micro- and nanoscale gold Hall sensors were fabricated, 
which facilitate accurate traceably calibrated scanning magnetic field microscopy (AFM-SHPM) at room temperature. The measurement data were in good agreement with simulation results, which underlines the reliability of the presented approach. The gold sensors exhibit a sensitivity of $3.2 \mathrm{mV} \mathrm{A}^{-1} \mathrm{~T}^{-1}$ with high long-term stability. Also, Hall sensors out of epitaxial, zero-band gap, semi-metallic graphene (on $\mathrm{SiC}$ ) were fabricated and studied. In contrast to the metallic gold sensors, the graphene samples show an outstanding high sensitivity of $1700 \mathrm{~V} \mathrm{~A}^{-1} \mathrm{~T}^{-1}$ but low time stability. This suggests that by proper isolation of the graphene sensors from environmental influences (e.g., using hexagonal boron nitride or aluminum dioxide), a higher performance could be achieved. This, in addition to the implementation of the graphene Hall sensor into the AFM cantilever, is the subject of future studies. For the $5 \mu \mathrm{m}$ gold AFM-SHPM, the uncertainty budget of the entire room temperature measurement process was analyzed and determined to be $\pm(7 \mathrm{mT}+13 \%)$. This method enables a direct quantitative characterization of magnetic microstructures in ambient conditions with the capability of generating three-dimensional maps of the sample's out-of-plane stray fields within a range from milliteslas (mT) to a few teslas (T). We expect that by developing a suitable, low-noise electronic for the $50 \mathrm{~nm}$ gold Hall sensors, the spacial resolution can be further increased, which will enable the direct traceable characterization of magnetic nanostructures at room temperature. The fabrication process is scalable, thus in principle allowing for high volume sensor production. Finally, the AFM-SHPM is a nondestructive and robust method for both scientific research and industrial applications, e.g., quality control within industrial processes.

Data availability. Raw data underlying the figures are available upon request to the authors if required.

Author contributions. MG, AS and TW fabricated the Hall sensors on cantilevers. DMP and KP prepared the graphene samples. MG and SS performed the experiments and analyzed the data. MG, $\mathrm{AM}$ and SS conducted simulations. HWS supervised the research work. MG prepared the manuscript with contributions from all coauthors.

Competing interests. The authors declare that they have no conflict of interest.

Acknowledgements. We thank Felix Nording from the Institut für Elektrische Messtechnik und Grundlagen der Elektrotechnik of Technische Universität Braunschweig in Germany for performing the noise measurements. Manuela Gerken gratefully acknowledges the support of the Braunschweig International Graduate School of Metrology B-IGSM and the DFG Research Training Group 1952 Metrology for Complex Nanosystems. Davood Momeni Pakdehi acknowledges support from the School for Contacts in Nanosystems (NTH nano). The company Sensitec GmbH provided the test sample, the magnetic-scale SST250HFA-04.

Financial support. This research has been supported by the European Metrology Programme for Innovation and Research (grant no. 15SIB06), the European Metrology Programme for Innovation and Research (grant no. 18SIB07) and the Deutsche Forschungsgemeinschaft (DFG) (grant no. 390837967).

This open-access publication was funded by the Physikalisch-Technische Bundesanstalt.

Review statement. This paper was edited by Michael Kraft and reviewed by two anonymous referees.

\section{References}

Chenaud, B., Segovia-Mera, A., Delgard, A., Feltin, N., Hoffmann, A., Pascal, F., Zawadzki, W., Mailly, D., and Chaubet, C.: Sensitivity and noise of micro-Hall magnetic sensors based on InGaAs quantum wells, J. Appl. Phys., 119, 024501, https://doi.org/10.1063/1.4939288, 2016.

Ciuk, T., Stanczyk, B., Przyborowska, K., Czolak, D., Dobrowolski, A., Jagiello, J., Kaszub, W., Kozubal, M., Kozlowski, R., and Kaminski, P.: High-Temperature Hall Effect Sensor Based on Epitaxial Graphene on High-Purity Semiinsulating 4H-SiC, IEEE Trans. Electron. Devices, 66, 3134-3138, https://doi.org/10.1109/TED.2019.2915632, 2019.

Corte-León, H., Neu, V., Manzin, A., Barton, C., Tang, Y., Gerken, M., Klapetek, P., Schumacher, H. W., and Kazakova, O.: Comparison and Validation of Different Magnetic Force Microscopy Calibration Schemes, Small, 1906144, https://doi.org/10.1002/smll.201906144, 2020.

Costa, M., Gaspar, J., Ferreira, R., Paz, E., Fonseca, H., Martins, M., Cardoso, S., and Freitas, P. P.: Integration of Magnetoresistive Sensors With Atomic Force Microscopy Cantilevers for Scanning Magnetoresistance Microscopy Applications, IEEE Trans. Magn., 51, 1-4, https://doi.org/10.1109/TMAG.2015.2448612, 2015.

Dauber, J., Sagade, A. A., Oellers, M., Watanabe, K., Taniguchi, T., Neumaier, D., and Stampfer, C.: Ultrasensitive Hall sensors based on graphene encapsulated in hexagonal boron nitride, Appl. Phys. Lett., 106, 193501, https://doi.org/10.1063/1.4919897, 2015.

Dede, M., Akram, R., and Oral, A.: 3D scanning Hall probe microscopy with $700 \mathrm{~nm}$ resolution, Appl. Phys. Lett., 109, 182407, https://doi.org/10.1063/1.4966896, 2016.

Göktaş, O., Weber, J., Weis, J., and von Klitzing, K.: Alloyed ohmic contacts to two-dimensional electron system in AlGaAs/GaAs heterostructures down to submicron length scale, Phys. E. Low-dimensional Syst. Nanostructures, 40, 1579-1581, https://doi.org/10.1016/j.physe.2007.09.115, 2008.

Hicks, C. W., Luan, L., Moler, K. A., Zeldov, E., and Shtrikman, H.: Noise characteristics of $100 \mathrm{~nm}$ scale $\mathrm{GaAs} / \mathrm{Al}_{x} \mathrm{Ga}_{1-x} \mathrm{As}$ 
scanning Hall probes, Appl. Phys. Lett., 90, 133512, https://doi.org/10.1063/1.2717565, 2007.

Hu, X. K., Dai, G., Sievers, S., Neu, V., and Schumacher, H. W.: Uncertainty Propagation and Evaluation of Nano-Scale Stray Field in Quantitative Magnetic Force Microscopy Measurements, in: IEEE 2018 Conference on Precision Electromagnetic Measurements, 8-13 July 2018 in Paris, France, 1-2, 2018.

Huang, L., Zhang, Z., Chen, B., Ma, X., Zhong, H., and Peng, L.M.: Ultra-sensitive graphene Hall elements, Appl. Phys. Lett., 104, 183106, https://doi.org/10.1063/1.4875597, 2014.

Hug, H. J., Stiefel, B., van Schendel, P. J. A., Moser, A., Hofer, R., Martin, S., Güntherodt, H.-J., Porthun, S., Abelmann, L., Lodder, J. C., Bochi, G., and O'Handley, R. C.: Quantitative magnetic force microscopy on perpendicularly magnetized samples, J. Appl. Phys., 83, 5609-5620, https://doi.org/10.1063/1.367412, 1998.

Kabanov, Y., Zhukov, A., Zhukova, V., and Gonzalez, J.: Magnetic domain structure of wires studied by using the magnetooptical indicator film method, Appl. Phys. Lett., 87, 142507, https://doi.org/10.1063/1.2077854, 2005.

Kazakova, O., Puttock, R., Barton, C., Corte-León, H., Jaafar, M., Neu, V., and Asenjo, A.: Frontiers of magnetic force microscopy, J. Appl. Phys., 125, 060901, https://doi.org/10.1063/1.5050712, 2019.

Klauenberg, K., Wübbeler, G., Mickan, B., Harris, P., and Elster, C.: A tutorial on Bayesian Normal linear regression, Metrologia, 52, 878-892, https://doi.org/10.1088/0026-1394/52/6/878, 2015.

Kruskopf, M., Pakdehi, D. M., Pierz, K., Wundrack, S., Stosch, R., Dziomba, T., Götz, M., Baringhaus, J., Aprojanz, J., Tegenkamp, C., Lidzba, J., Seyller, T., Hohls, F., Ahlers, F. J., and Schumacher, H. W.: Comeback of epitaxial graphene for electronics: large-area growth of bilayer-free graphene on $\mathrm{SiC}, 2 \mathrm{D}$ Mater., 3, 041002, https://doi.org/10.1088/2053-1583/3/4/041002, 2016.

Manzin, A. and Nabaei, V.: Modelling of micro-Hall sensors for magnetization imaging, J. Appl. Phys., 115, 17E506, https://doi.org/10.1063/1.4862090, 2014.

McCord, J.: Progress in magnetic domain observation by advanced magneto-optical microscopy, J. Phys. D. Appl. Phys., 48, 333001, https://doi.org/10.1088/0022-3727/48/33/333001, 2015.

Momeni Pakdehi, D., Aprojanz, J., Sinterhauf, A., Pierz, K., Kruskopf, M., Willke, P., Baringhaus, J., Stöckmann, J. P., Traeger, G. A., Hohls, F., Tegenkamp, C., Wenderoth, M., Ahlers, F. J., and Schumacher, H. W.: Minimum Resistance Anisotropy of Epitaxial Graphene on SiC, ACS Appl. Mater. and Interfaces, 10, 6039-6045, https://doi.org/10.1021/acsami.7b18641, 2018.

Momeni Pakdehi, D., Pierz, K., Wundrack, S., Aprojanz, J., Nguyen, T. T. N., Dziomba, T., Hohls, F., Bakin, A., Stosch, R., Tegenkamp, C., Ahlers, F. J., and Schumacher, H. W.: Homogeneous Large-Area Quasi-Free-Standing Monolayer and Bilayer Graphene on SiC, ACS Appl. Nano Mater., 2, 844-852, https://doi.org/10.1021/acsanm.8b02093, 2019.

Nabaei, V., Rajkumar, R. K., Manzin, A., Kazakova, O., and Tzalenchuk, A.: Optimization of Hall bar response to localized magnetic and electric fields, J. Appl. Phys., 113, 064504, https://doi.org/10.1063/1.4790508, 2013.

Nagashio, K., Nishimura, T., Kita, K., and Toriumi, A.: Contact resistivity and current flow path at metal/graphene contact, Appl. Phys. Lett., 97, 143514, https://doi.org/10.1063/1.3491804, 2010 .
NanoMagnetics Instruments: micro-Hall Probes \& microHall Probe Gaussmeter, available at: https://www. nanomagnetics-inst.com (last access: 26 July 2019), 2017a.

NanoMagnetics Instruments: RT-SHPM Room Temperature Scanning Hall Probe Microscope, available at: https://www. nanomagnetics-inst.com (last access: 26 July 2019), 2017b.

Nording, F., Weber, S., Ludwig, F., and Schilling, M.: Measurement system for temperature dependent noise characterization of magnetoresistive sensors, Rev. Sci. Instrum., 88, 35006, https://doi.org/10.1063/1.4978324, 2017.

Nording, F. M.: Messsystem zur Rauschanalyse von magnetoresistiven Sensoren, $\mathrm{PhD}$ thesis, Technische Universität Braunschweig, Mensch und Buch Verlag, Berlin, https://doi.org/10.24355/dbbs.084-201905080926-0, 2019.

Novoselov, K. S., Morozov, S. V, Dubonos, S. V, Missous, M., Volkov, A. O., Christian, D. A., and Geim, A. K.: Submicron probes for Hall magnetometry over the extended temperature range from helium to room temperature, J. Appl. Phys., 93, 10053-10057, https://doi.org/10.1063/1.1576492, 2003.

Pross, A., Crisan, A. I., Bending, S. J., Mosser, V., and Konczykowski, M.: Second-generation quantum-well sensors for room-temperature scanning Hall probe microscopy, J. Appl. Phys., 97, 096105, https://doi.org/10.1063/1.1887828, 2005.

Sandhu, A., Kurosawa, K., Dede, M., and Oral, A.: 50 nm Hall Sensors for Room Temperature Scanning Hall Probe Microscopy, Jpn. J. Appl. Phys., 43, 777-778, https://doi.org/10.1143/JJAP.43.777, 2004.

Shaw, G., Kramer, K., Dempsey, N. M., Hasselbach, K., Kramer, R. B. G., Dempsey, N. M., and Hasselbach, K.: A Scanning Hall Probe Microscope for high resolution, large area, variable height Magnetic Field Imaging, Rev. Sci. Instrum., 87, 113702, https://doi.org/10.1063/1.4967235, 2016.

Sonusen, S., Karci, O., Dede, M., Aksoy, S., and Oral, A.: Single layer graphene Hall sensors for scanning Hall probe microscopy (SHPM) in 3-300K temperature range, Appl. Surf. Sci., 308, 414-418, https://doi.org/10.1016/j.apsusc.2014.04.191, 2014.

Takezaki, T. and Sueoka, K.: Quantitative current measurements using scanning magnetoresistance microscopy, Ultramicroscopy, 108, 970-974, https://doi.org/10.1016/j.ultramic.2008.03.007, 2008.

Tang, C.-C., Li, M.-Y., Li, L. J., Chi, C. C., and Chen, J. C.: Characteristics of a sensitive micro-Hall probe fabricated on chemical vapor deposited graphene over the temperature range from liquid-helium to room temperature, Appl. Phys. Lett., 99, 112107, https://doi.org/10.1063/1.3640218, 2011.

van Schendel, P. J. A., Hug, H. J., Stiefel, B., Martin, S., and Güntherodt, H.-J.: A method for the calibration of magnetic force microscopy tips, J. Appl. Phys., 88, 435-445, https://doi.org/10.1063/1.373678, 2000.

Weis, J. and von Klitzing, K.: Metrology and microscopic picture of the integer quantum Hall effect, Philos. Trans. R. Soc. A Math. Phys. Eng. Sci., 369, 3954-3974, https://doi.org/10.1098/rsta.2011.0198, 2011. 